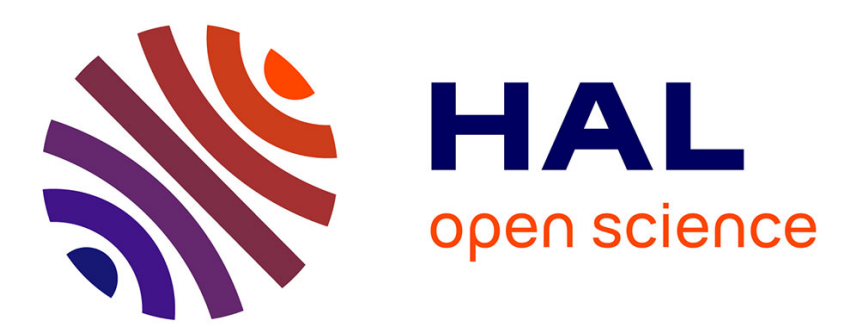

\title{
Couches minces de grenat substitué déposées par le procédé pyrosol pour l'enregistrement magnéto-optique
}

J. Deschanvres, J. Joubert

\section{To cite this version:}

J. Deschanvres, J. Joubert. Couches minces de grenat substitué déposées par le procédé pyrosol pour l'enregistrement magnéto-optique. Journal de Physique IV Proceedings, 1992, 02 (C3), pp.C3-29-C333. 10.1051/jp4:1992304 . jpa-00251509

\section{HAL Id: jpa-00251509 https://hal.science/jpa-00251509}

Submitted on 1 Jan 1992

HAL is a multi-disciplinary open access archive for the deposit and dissemination of scientific research documents, whether they are published or not. The documents may come from teaching and research institutions in France or abroad, or from public or private research centers.
L'archive ouverte pluridisciplinaire HAL, est destinée au dépôt et à la diffusion de documents scientifiques de niveau recherche, publiés ou non, émanant des établissements d'enseignement et de recherche français ou étrangers, des laboratoires publics ou privés. 


\title{
Couches minces de grenat substitué déposées par le procédé pyrosol pour l'enregistrement magnéto-optique
}

\author{
J.L. DESCHANVRES et J.C. JOUBERT
}

Laboratoire des Matériaux et du Génie Physique, URA 1109 CNRS, Ecole Nationale Supérieure de Physique de Grenoble, BP. 46, 38402 Saint Martin d'Heres, France

\begin{abstract}
For magneto-optic memories application BiDyGaIG thin films $w$,re grown on glass substrates by an aerosol chemical vapor deposition process. After annealing the films behave a non textured polycrystalline garnet phase. The Faraday hysteresis loops measured at $\lambda=633 \mathrm{~nm}$, exhibited a good squareness with a coercive field higher than 1000E and a Faraday rotation higher than $2 \%$ m. By static recording it has been possible to write small domains of 2 to $15 \mu \mathrm{m}$.

RESUME

De par leurs propriétés magnéto-optiques et leur très bonne résistance à la corrosion.les couches minces de grenat substitué ( $\mathrm{BiDy}$ ) 3 ( $\mathrm{FeGa}$ ) $5 \mathrm{O}_{12}$ sont candidates potentielles pour les média magnéto-optiques de deuxième génération. Pour réaliser de telles couches nous développons dans notre laboratoire un procédé chimique peu coûteux, le procédé pyrosol, qui est basé sur la pyrolyse d'un aérosol contenant des précurseurs organométalliques. Les couches, d'une épaisseur de $2000 \AA$ a $5000 \AA$ déposées sur des substrats de verre a $515^{\circ} \mathrm{C}$, sont parfaitement transparentes et adhérentes, et font apparaître après recuit en diffraction des rayons $\mathrm{X}$ une structure polycristalline non orientée. Un taux de substitution de 2 bismuth par unité de formule permet d'obtenir un effet Faraday supérieur a $2 \% / \mathrm{m}$ pour une longueur d'onde de $632,8 \mathrm{~nm}$ et des tests d'écriture thermomagnétique par laser ont permis d'inscrire des domaines d'un diamètre inférieur à $2 \mu \mathrm{m}$.
\end{abstract}

\section{INTRODUCTION}

Pour la seconde génération des média magnéto-optiques, la course à l'augmentation de la densité de stockage conduira à travailler dans le bleu. A ces longueurs d'ondes les couches minces de grenat substitué au bismuth présentent les effets magnéto-optiques les plus importants et la résistance à la corrosion de ces couches d'oxyde renforce l'interêt de leur utilisation en tant que média. En plus du bismuth les différentes substitutions font intervenir le dysprosium, qui contribue à l'obtention de l'anisotropie perpendiculaire par l'augmentation du coefficient de magnétostriction, et le gallium,qui contibue à diminuer la température de Curie afin de faciliter le processus d'écriture thermomagnétique. Aujourd'hui plusieurs laboratoires dans le monde mènent des études sur ce sujet à partir en particulier de technique de dépôt par pulvérisation cathodique [1,2] ou par une voie de pyrolyse de solution de nitrates [3].Au sein de notre laboratoire, dans la suite de travaux sur les ferrites spinelles [4], nous développons un procédé chimique peu coûteux, le procédé pyrosol, qui est basé sur la pyrolyse d'un aérosol contenant des précurseurs organométalliques. Ce procédé permet d'obtenir des dépôts bien adhérents et transparents avec en particulier la croissance épitaxiale de couche de YIG [5].

\section{ETUDE DE LA CINETIQUE DE DEPOT}

La technique de dépôt a été décrite précédement [6]. Le processus peut se résumer en trois phases avec tout d'abord la production d'un brouillard par pulvérisation ultrasonore à partir d'une solution contenant les précurseurs organométalliques. Le brouillard est ensuite 
entrainé vers la zone de dépôt ou il vient se pyrolyser sur le substrat chauffé. Pour réaliser les dépôts nous utilisons une solution de butanol contenant comme précurseurs organométalliques:

- L'acétylacétonate de fer $=\mathrm{Fe}(\mathrm{AA})_{3}=\mathrm{Fe}\left(\mathrm{C}_{5} \mathrm{H}_{7} \mathrm{O}_{2}\right)_{3}$

- L'acétylacétonate de gallium $=\mathrm{Ga}(\mathrm{AA})_{3}=\mathrm{Ga}\left(\mathrm{C}_{5} \mathrm{H}_{7} \mathrm{O}_{2}\right)_{3}$

- Le tétraméthylheptanédionate de dysprosium $=\mathrm{Dy}(\mathrm{TMHD}) 3=\mathrm{Dy}\left(\mathrm{C}_{11} \mathrm{H}_{\left.19 \mathrm{O}_{2}\right)}\right)_{3}$

- Le triphenyl bismuth $=\mathrm{Bi}(\mathrm{Ph}) 3=\mathrm{Bi}\left(\mathrm{C}_{6} \mathrm{H}_{5}\right) 3$

L'étude de l'influence de la température sur la cinétique de réaction de chacun des précurseurs nous permet d'obtenir une composition contrôlée. Cette étude est basée sur la comparaison entre la composition de la couche mince déposée, mesurée par micro-analyse $\mathbf{X}$ à l'aide d'un programme spécifique pour couches minces [7], et la composition de la solution, ceci pour différentes solutions et température de dépôt. La figure 1 montre qu'entre $500^{\circ} \mathrm{C}$ et $530^{\circ} \mathrm{C}$ la cinétique de réaction de l'acétylacétonate de fer et de gallium varie de la même façon avec la temperature.Ainsi la proportion de gallium par rapport au fer dans la couche peut être contrôlée par la composition de la solution comme l'indique la figure 2. Au contraire dans le même intervalle, la cinétique de réaction du triphenyl bismuth est nettement plus activée par la température que celle des autres précurseurs. En effet comme le montre la figure 3, la proportion du bismuth par rapport au dysprosium dans la couche augmente avec la température

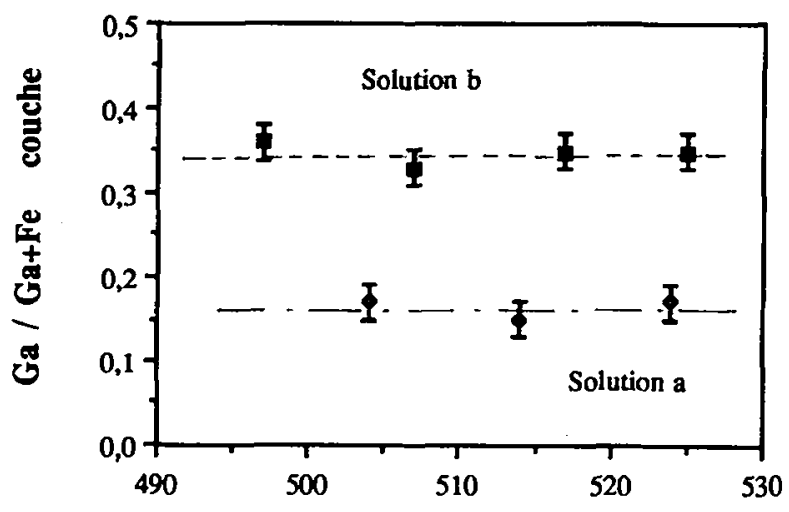

TEMPERATURE ${ }^{\circ} \mathrm{C}$

Fig. 1: Taux de substitution du gallium $(\mathrm{Ga} / \mathrm{Ga}+\mathrm{Fe})$ dans la couche en fonction de la température de dépôt pour deux solutions différentes.

-(a) Dy(TMHD)3- 12; $\mathrm{Bi}(\mathrm{Ph}) 3-8 ; \mathrm{Fe}(\mathrm{AA}) 3-5 ; \mathrm{Ga}(\mathrm{AA}) 3-510^{-3} \mathrm{Mol} / \mathrm{L}$

-(b) Dy(TMHD) 3-10; Bi(Ph)3-10; Fe(AA)3-7.2; Ga(AA)3-2.3 10-3 Mol/L $A A=$ acetylacetonate $\quad P h=$ phenyl

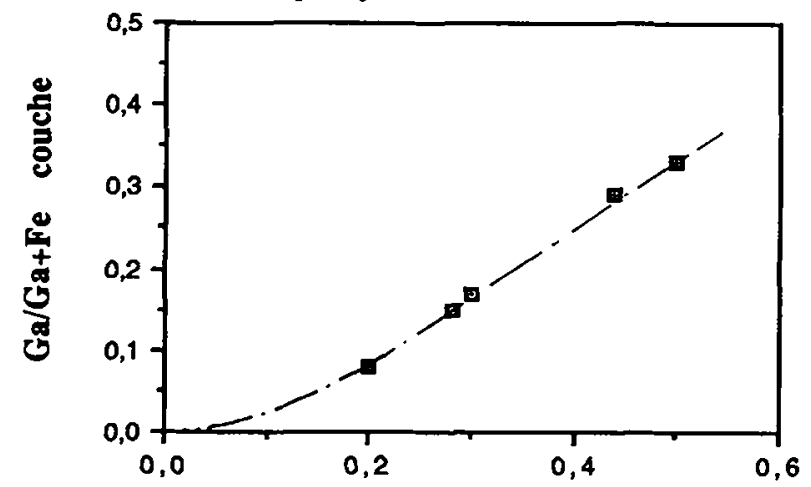

$\mathrm{Ga} / \mathrm{Ga}+\mathbf{F e}$ solution

Fig. 2: Taux de substitution du gallium $(\mathrm{Ga} / \mathrm{Ga}+\mathrm{Fe})$ dans la couche en fonction $\mathrm{du}$ même rapport dans la solution. 


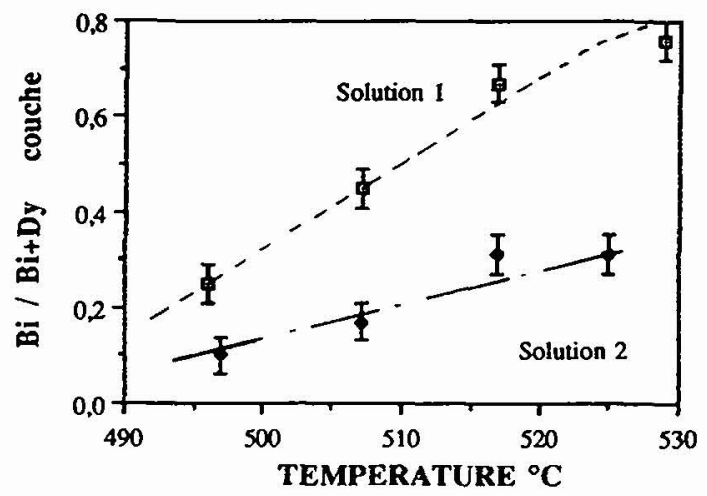

Fig. 3: Taux de substitution du bismuth (Bi/Bi+Dy) dans la couche en fonction de la température de dépôt pour deux solutions différentes.
-(1) Dy(TMHD)3- 9 ; Bi(Ph)3-11; Fe(AA)3- 5 ; Ga(AA)3- $510^{-3} \mathrm{Mol} / \mathrm{L}$
-(2) Dy(TMHD) $3-12 ; \mathrm{Bi}(\mathrm{Ph}) 3-8 ; \mathrm{Fe}(\mathrm{AA}) 3-5 ; \mathrm{Ga}(\mathrm{AA}) 3-510^{-3} \mathrm{Mol} / \mathrm{L}$

\section{ETUDES CRISTALLOGRAPHIQUE ET MORPHOLOGIQUE}

Les couches déposées sont amorphes, transparentes et bien adhérentes. Après un recuit entre $600^{\circ} \mathrm{C}$ et $650^{\circ} \mathrm{C}$ pendant 3 heures, elles cristallisent sous la forme d'une phase grenat non orientée. La température de cristallisation décroit avec l'augmentation du taux de bismuth [8]. Le taux maximum de substitution est de 2.1 bismuth par unité de formule. Mais au delà d'un taux de 2 , comme le montre l'étude en microscopie électronique à balayage, les couches présentent une morphologie inhomogène avec l'apparition de gros grains (fig.4).
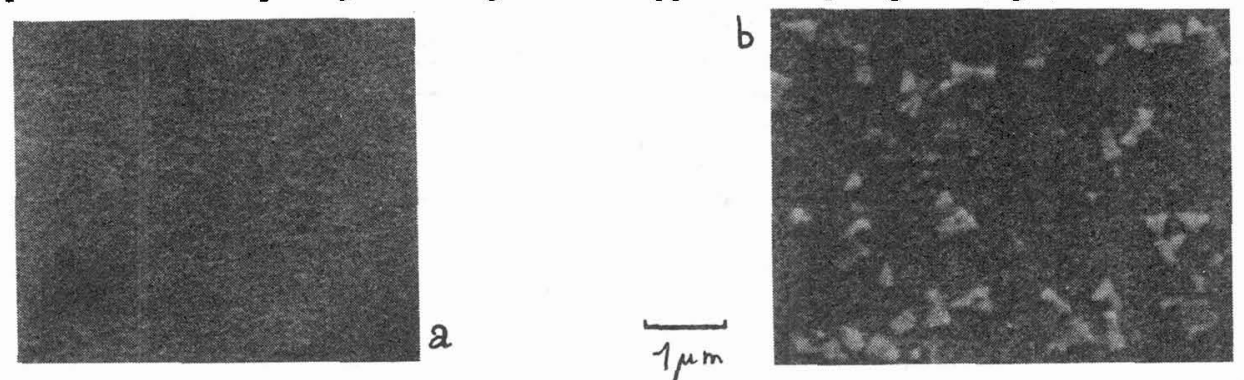

Fig. 4: Clichés de microscopie électronique à balayage de couches recuites à $650^{\circ} \mathrm{C}$ pendant 3 heures et réalisées avec deux taux de substitution du bismuth différents:

(a) Bi 1 Dy 2 Fe4.2 Ga0.8 $\mathrm{O}_{12}$

(b) Bi 2 Dy 1 Fe4.2 $\mathrm{Ga}_{0.8} \mathrm{O}_{12}$

\section{ETUDES DES CARACTERISTIQUES MAGNETO-OPTIQUES}

Les mesures des propriétés magnétooptiques ont été réalisées à l'aide d'un banc Faraday travaillant dans le rouge $(\lambda=633 \mathrm{~nm})$. Les cycles Faraday obtenus montrent que les couches réalisées présentent de bonnes caractéristiques avec des cycles bien rectangulaires (fig.5a) et un champ coercitif supérieur à 10000E. Un taux de substitution de 2 bismuth par unité de formule permet d'obtenir un effet Faraday supérieur à $2 \%$ m. L'observation de cycles inversés (fig.5b) pour certaines couches ainsi que l'étude de l'évolution des cycles Faraday en fonction de la température de mesure ont mis en évidence l'effet de la température de compensation. La rotation Faraday est inversée suivant que la température lors de la mesure du cycle est inférieure ou supérieure à la temperrature de compensation. Celle ci diminue quand le taux de bismuth 
augmente,i.e. quand le nombre de dysprosium diminue (Tab.I). Au voisinage de la température de compensation le champ coercitif diverge vers l'infini (fig 6). L'obtention d'une temperature de compensation entre 50 et $100^{\circ} \mathrm{C}$ permet d'avoir des champs coercitifs élevés à la température ambiante et une variation rapide du champ coercitif au dessus de la température de compensation jusqu'au point de Curie. La valeur de la température de compensation dépend de la composition et des recuits thermiques qui modifient la répartition des cations sur les différents sites.

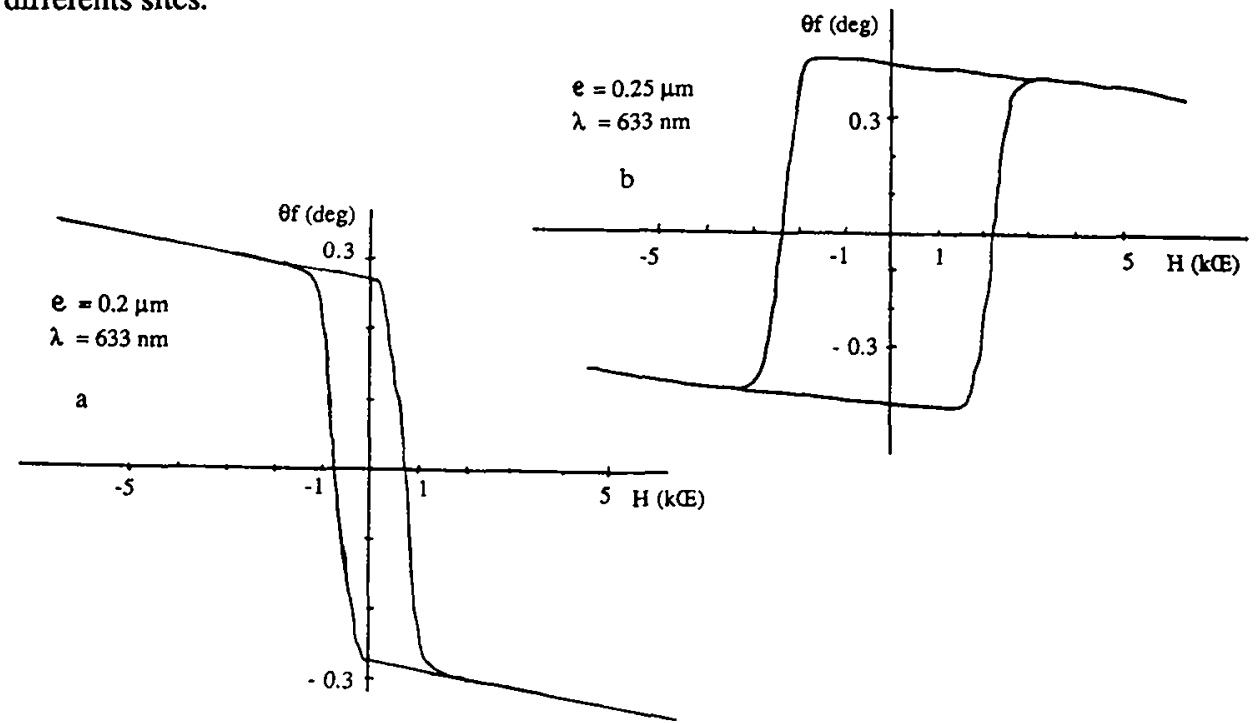

Fig. 5: Cycles Faraday de deux couches de compositions différentes, mesurés à $\lambda=633 \mathrm{~nm}$.

(a) Bi1.6 Dy $1.4 \mathrm{Fe}_{4.2} \mathrm{Ga0} .8 \mathrm{O}_{12}$ et recuite à $650^{\circ} \mathrm{C}$.

(b) Bi 2 Dy $1 \quad \mathrm{Fe}_{4.2} \mathrm{Ga}_{0.8} \mathrm{O}_{12}$ et recuite à $600^{\circ} \mathrm{C}$.

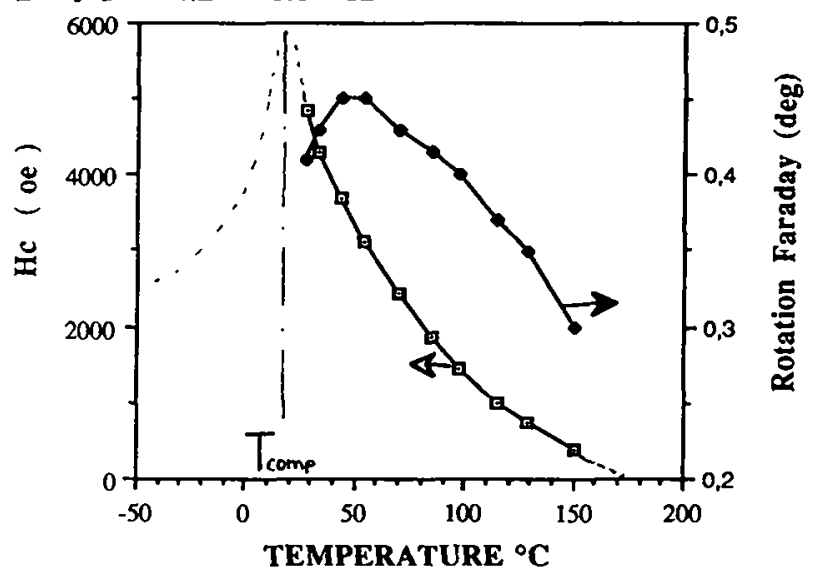

Fig. 6: : Variation, en fonction de la temperature, du champ coercitif et de la rotation Faraday pour une couche de composition $\mathrm{Bi}_{2} \mathrm{Dy}_{1} \mathrm{Fe}_{4.2}$ Ga0.8 $\mathrm{O}_{12}$ et d'une épaisseur de $250 \mathrm{~nm}$. Cette variation est déduite de la mesure des cycles Faraday pour différentes températures.

TABLE I: Temperature de compensation et de Curie

Composition

$\mathrm{Bi}_{2} \mathrm{Dy}_{1} \mathrm{Fe}_{4.2} \mathrm{Ga}_{0.8} \mathrm{O}_{12}$

$\mathrm{Bi}_{1.9} \mathrm{Dy}_{1.1} \mathrm{Fe}_{4.2} \mathrm{Ga}_{0.8} \mathrm{O}_{12}$

$\mathrm{Bi}_{1.1} \mathrm{Dy}_{1.9} \mathrm{Fe}_{4.2} \mathrm{Ga}_{0.8} \mathrm{O}_{12}$
Tcompensation

$<$ t.a.

$58^{\circ} \mathrm{C}$

$77^{\circ} \mathrm{C}$
Tcurie

$175^{\circ} \mathrm{C}$

$180^{\circ} \mathrm{C}$

$180^{\circ} \mathrm{C}$ 


\section{TEST D'ECRITURE}

Pour réaliser les test les films ont été recouverts d'une couche de chrome qui joue un rôle de réflecteur et un rôle d'absorbant thermique. L'inscription a été réalisée avec une diode laser à $\lambda=830 \mathrm{~nm}$ ayant une puissance de $10 \mathrm{~mW}$. La photo $\mathrm{n}^{\circ} 7$ réalisée en microscopie laser à balayage, montre les domaines magnétiques inscrits pour différentes durées d'impulsion. La forme des domaines est bien régulière et leur taille varie entre 1 et $15 \mu \mathrm{m}$ en fonction de la durée de l'impulsion laser.

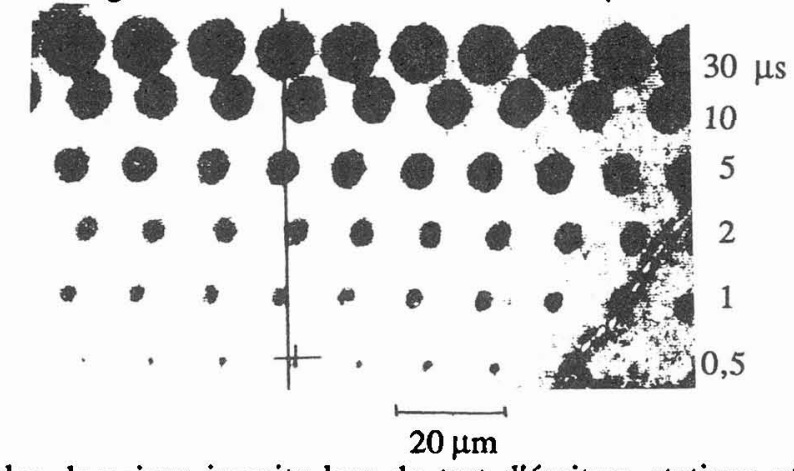

Fig. 7: Observation des domaines inscrits lors de test d'écriture statique réalisés avec différentes durées d'impulsion laser.

\section{REMERCIEMENTS}

L'ensemble des caractérisations magnéto-optiques ont été réalisées au LETI dans le groupe de Mr Béchevet et Mr Daval que je remercie pour leur aide précieuse.

\section{CONCLUSION}

Par l'utilisation de précurseurs organométalliques de la famille des $\beta$ dicétones et du triphenyl bismuth, il a été possible d'élaborer par le procédé pyrosol des couches de grenat substitué de composition $\left(\mathrm{Bi}_{\mathrm{x}} \mathrm{Dy}_{3-\mathrm{x}}\right)\left(\mathrm{Fe}_{5-\mathrm{y}} \mathrm{Ga}_{\mathrm{y}}\right) \mathrm{O}_{12}$ avec $\mathrm{x}$ variant entre 0,5 et 2 et $\mathrm{y}$ variant entre 0,2 et 1,5 . Les couches après recuit présentent de bonnes caractéristiques avec un champ coercitif supérieur à 1000 Oe et une rotation Faraday supérieure à $2 \% / \mu \mathrm{m}$. A l'avenir l'obtention d'un bon rapport signal sur bruit lors de la lecture des domaines requiert la limitation du phénomène de diffusion de la lumière par les joints de grains. Cette limitation doit être atteinte par la réduction de la taille des grains à un diamètre inférieure à $500 \AA$ soit en optimisant le processus de recuit soit en intégrant au dépôt des substituants qui limiteraient la croissance des grains.

\section{REFERENCES}

[1] M. Abe et M. Gomi, Advances in magnetooptics, J. Magn. Soc. Jpn. Vol 11, Suppl. NoS1, (1987) 299.

[2] D. Challeton, J. Mouchot, B. Béchevet et col. , MORIS'91, J. Magn. Soc. Jpn. Vol 15, Suppl. NS1, (1991) 226.

[3] K. Nakagawa, K. Odagawa, A. Itoh, Jpn. J. Appl. Phys., Vol 29, N99, (1990) 1690.

[4] J.L. Deschanvres, M. Langlet, JMMM. (1990) 437-438.

[5] J.L. Deschanvres, M. Langlet,, J.C. Joubert, IEEE Trans. Mag. Vol 26 n $^{\circ} 1$, (1990) 187

[6] J.L. Deschanvres,F. Cellier,M. Labeau, M. Langlet et J.C. Joubert J. Phys.,Tome 50, mai 1989, C5 695-705.

[7] J.L. Pouchou et F. Pichoir, La Recherche Aérospatiale N5, (1984) 349-367.

[8] J.L. Deschanvres, M. Langlet, J.C. Joubert, JMMM. Vol 101 (1991)224 